links the antibody to the phage instead of the antibody being an integral part of one of the coat proteins. This makes it easier to separate the antibody at a later stage. MorphoSys also offers 'Antibodies by design', a service that will develop recombinant monoclonal HuCAL antibodies for individual targets. "This is especially interesting for researchers in industry and academia, as the turnaround time is only about two months and the cost starts at about E5,000 [US\$6,000]," says Claudia Gutjahr-Loeser of MorphoSys.

Rather than going for comprehensiveness, a human-antibody library can be biased in favour of particular targets, for example by taking the initial antibody coding sequences from individuals vaccinated against a particular disease. Blood from soldiers immunized against anthrax was the starting point for a Fab antibody library that has allowed Katherine Bowdish and her

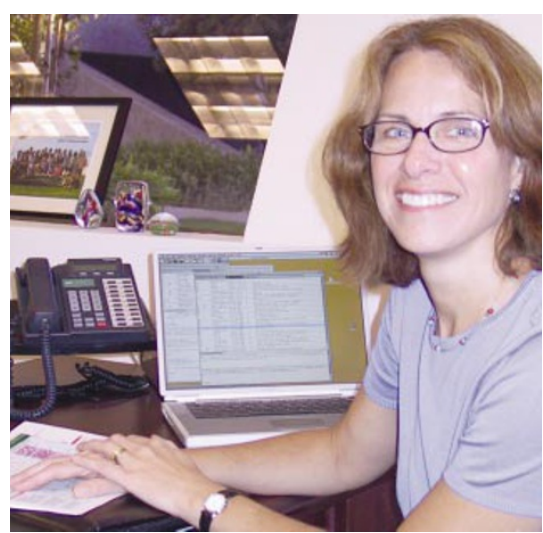

Katherine Bowdish: getting to grips with anthrax. colleagues at Alexion Antibody Technologies in San Diego, California, to identify highaffinity human monoclonal antibodies to critical elements of the anthrax toxin (M. A. Wild et al. Nature Biotechnol. 21, 1305-1306; 2003). The technology is also applicable to diseases such as smallpox, says Bowdish.

As the source of its library, Affitech in Oslo, Norway, also uses blood from selected donors known to produce antibodies of interest, such as cancer patients, or people who have been vaccinated or are recovering from an infectious disease. Affitech's chief executive Martin Welschof says that this, combined with the company's proprietary screening system, avoids the need for repetitive rounds of selection and means that it can move from a $10-\mathrm{ml}$ blood sample to a highaffinity human monoclonal antibody of the desired specificity within four weeks.

\section{Small is beautiful}

If a therapeutic antibody has to penetrate far into body tissues, or into a tumour, for example, smaller may well be better. Although some companies are concentrating on scFv antibody fragments, UK-based Domantis is producing even smaller human antibodies from its phage libraries: these consist of just a single variable domain.

Like scFv fragments, these 'domain antibodies' can be mass-produced relatively cheaply in Escherichia coli or yeast. They are also very stable, which opens up new options for therapy, such as a powder that can be inhaled to treat asthma and other lung diseases, or a tablet to be taken orally for gut inflammation. "We're only going after areas where domain antibodies have unique

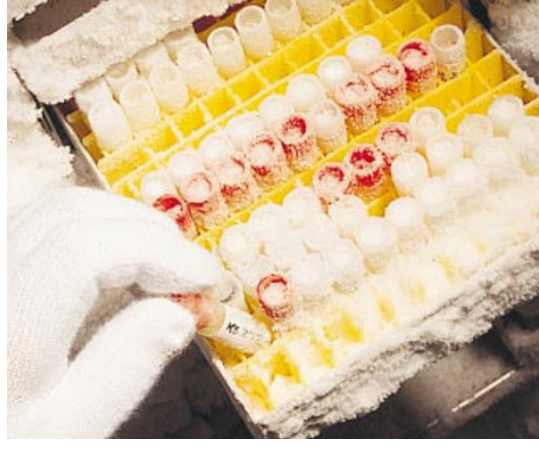

Frozen phage: CAT's library in store.

advantages," says Ian Tomlinson, co-founder and chief scientific officer of Domantis. In partnership with Peptech in North Ryde, New South Wales, Australia, Domantis is developing a domain antibody against TNF$\alpha$ for the treatment of rheumatoid arthritis and other inflammatory diseases.

Part of the inspiration for pursuing domain antibodies was the natural domain antibodies found in camels, dromedaries, llamas - and sharks. These natural antibodies are also being explored for their potential applications by start-up biopharmaceutical company Ablynx of Zwijnaarde, Belgium.

\section{Mouse power}

Both Abgenix of Fremont, California, and Medarex of Princeton, New Jersey, take a different approach to making large numbers of different human antibodies for screening - they get mice to do it for them. In Abgenix's XenoMouse and Medarex's HuMab-Mouse, murine germ-line antibody genes have been replaced with human ones.

\title{
ANTIBODIES WHERE YOU WANT THEM
}

Antibodies are normally built inside cells, take up their final form as they are secreted from the cell, and function exclusively outside cells, binding to cell surfaces and extracellular molecules. But Terry Rabbitts at the MRC Laboratory of Molecular Biology in Cambridge, UK, wants to use antibodies' highly specific binding properties inside the cell itself. He has engineered cells to make fragments consisting of just a heavy-chain variable domain and hold them within the cell. These single-domain antibodies can interact with intracellular proteins at binding affinities of 10 nanomolar or better. At Iclectus, an MRC spin-off company based in London, Rabbitts' colleagues are developing these fragments as intracellular antibodies or 'intrabodies'.

They became interested in intrabodies while they were unravelling the role of transcription factors involved in producing chromosomal translocations in tumour cells. Using intrabodies to bind and block these transcription factors inside the cell opened up a new range of research possibilities as well as pointing the way to new anticancer therapies. Transcription factors operate by binding other proteins, so "if you have an

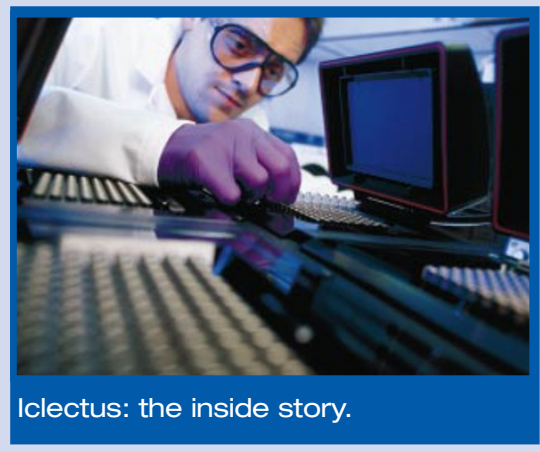

intrabody that has an affinity close to or better than the affinity of the interacting partner, you should be able to block the interaction using the intrabody", says Rabbitts.

Intrabodies offer the cell biologist the prospect of highly specific tools that can probe protein-protein interactions in situ more delicately than techniques that simply remove one of the proteins from the scene by antisense or gene knockout. Because an intrabody binds to a specific site on a protein, it can block a protein's interaction with one partner while allowing it to continue acting with another.

Another use is for the validation of potential drug targets, one of the first and vital steps in drug discovery. Most potential target proteins are modular, so intrabodies that bind to and block the function of individual modules could help to determine which part of the protein is key to causing the disease.

Placing localization signals on an intrabody allows it to be directed to various parts of the cell, such as the nucleus. "With intrabodies you can be more versatile in your experiments and ask more questions than, say, with RNA interference," says Rabbitts. 